\title{
Variation of main components according to the number of steaming and drying of Rehmanniae radix preparata
}

\author{
Ui Joung Youn ${ }^{1}$, Bon-Seok Gu ${ }^{2,3}$, Kyung Hee Kim, ${ }^{1,3}$ Chulgyu $\mathrm{Ha}^{4}$ and In Chan Jung ${ }^{3^{*}}$
}

${ }^{1}$ Division of life Sciences, Korea Polar Research Institute, Inchon, Republic of Korea

${ }^{2}$ Agricultural Corporation Aram Limited, Seosan, Republic of Korea

${ }^{3}$ Department of Chemistry, Hanseo University, Seosan, Republic of Korea

${ }^{4}$ Department of Bioprocess Technology, Biocampus of Korea Polytechnic, Republic of Korea

\section{Key Words}

catalpol, Rehmanniae Radix, impregnation method, 5-HMF.

\begin{abstract}
Contents of compounds in Rehmanniae Radix change depending on the number of steaming and drying and the drying method. In this study, as an impregnation method for dried Rehmanniae Radix, takju impregnation and cheongju impregnation were carried out and steaming and drying were repeated for 9 times. The changes of 5-HMF and catalpol contents were analyzed according to the number of repetition times to investigate which stage of steaming and drying is preferable. Also, total nitrogen, crude fat, ash, and crude fiber were measured to analyze changes in general components. 5-HMF was not detected in dried Rehmanniae Radix. As a result of repetitive steaming and drying, the content of 5-HMF increased only slightly from 1 to 4 -times steaming and drying but increased significantly from 5-times. The catalpol in dried Rehmanniae Radix was not detected after 5 times of steaming and drying. Sucrose, maltose, and glucose were included in dried Rehmanniae Radix before steaming and drying. However, after the process in both Takju impregnation and Cheongju impregnation, galactose and fructose tended to decrease after production and sucrose and glucose tended to
\end{abstract}

Received: May 3, 2018 Reviewed: June 4, 2018 Accepted: June 12, 2018

(c) This is an Open-Access article distributed under the terms of the Creative Common Attribution Non-Commercial License (http://creativecommons.org/licenses/by-nc/4.0/) which permits unrestricted noncommercial use, distribution, and reproduction in any medium, provided the original work is properly cited.

(2) This paper meets the requirements of KS X ISO 9706, ISO 9706-1994 and ANSI/NISO Z39.48-1992 (Permanence of Paper). decrease after the increase. In this study condition, 6 -times and more steaming and drying were appropriate process which met the content criteria (not less than $0.1 \%$ ) of the Korean Pharmacopoeia (8th edition) for 5-HMF, an index component for quality control of Rehmanniae Radix Preparata.

\section{Introduction}

The botanical names of Scrophulariaceae include Rehmannia glutinosa Libosch, Rehmannia glutinosa Libosch var. purpurea Makino, Rehmannia, glutinosa Libosch var. hueichingensis(Chao et Schih), Hsiao, etc[1-2]. Rehmanniae radix is a perennial plant and the length of its aerial part is approximately $20-30 \mathrm{~cm}$. The length of roots is about $30 \mathrm{~cm}$. The part right below where sclerophyll comes out is thin but becomes thicker and thicker as it goes down, but it gets thinner again near the end. Its diverse varieties have different shades including lemon yellow, dark yellow, and red yellow. The outer part of the cross-section is creamywhite, while the inside is ivory-white. In South Korea, the domestic growing method for Rehmanniae radix changed from native species to clone selection in 1999. The domestic variety of Rehmanniae radix has many thick roots and has a high content of active ingredients and a high processing yield into Rehmanniae radix preparata. The major ingredients of Rehmanniae radix include $\beta$-sitosterol, mannitol, catalpol, stimastenol, camphesterol, rehmannin, alkaloids, fatty acid, glu- 
cose, vitamin A. The pharmacological action is the dilatation and contraction of blood vessels and the acceleration of blood coagulation.

It is used for symptoms including cardiac diuresis, blood sugar drop. antipyretic, and a disease symptomized by thirst. Raw Rehmanniae radix is harvested in spring or fall and can be used without any processing. After cleaning it with water, peel it off with a bamboo knife or a plastic brush and dry it at a temperature condition of $40-50^{\circ} \mathrm{C}$ to produce dried Rehmanniae radix. The weight of dried Rehmanniae radix is approximately $25-30 \%$ of raw Rehmanniae radix. Depending on drying methods, Rehmanniae radix can be classified as raw Rehmanniae radix, dried Rehmanniae radix, or Rehmanniae radix preparata. Rehmanniae radix preparata is produced by steaming and drying raw Rehmanniae radix and dried Rehmanniae radix and is used for clinical purposes[3]. According to the 7 th revised edition of the Korean Pharmacopoeia, dried Rehmanniae radix preparata is required to have the content of 5-hydroxymethyl-2-furaldehyde $\left(\mathrm{C}_{6} \mathrm{H}_{6} \mathrm{O}_{3}: 126.11\right)$ at higher than $0.1 \%$, and is subject to quality management. The production method of Rehmanniae radix preparata is to immerse cleanly-washed Rehmanniae radix in wine, take it out, steam it, and dry it under the sun, and the process of steaming and drying is repeated for nine times. Unlike raw or dry Rehmanniae radix's fever alleviating and hemostatic effects, Rehmanniae radix preparata has tonic and calming effects. Therefore, depending on processing methods, its pharmacological action and compounds can be changed[4-5]. Among experimental studies on Rehmanniae radix, a study by Jeong (2001) reported that Rehmanniae radix preparata plays an important role in the early differentiation of bone marrow cells[6], and another study by Chang et al. (2007) reported that since Rehmanniae radix preparata has the effect of improving the survival rate of GC-1 cell, the male gem cell, it can be used for treatment of male infertility[7]. Also, a study by Lee et al. [8] reported that Rehmanniae radix preparata that are steamed for nine times and dried for nine times can normalize an increased blood-lipid content rate induced by hyperlipidemia and reduce the number of free radicals increased by hyperlipidemia.

Although there has been much interest in and many studies and research findings on various effects of Rehmanniae radix preparata on physiological activation, there have been not enough studies to exactly show how steaming and drying temperatures can affect the variation of contents[9-10]. Therefore, this study used dried Rehmanniae radix and repeated the process of steaming and drying at a precise temperature under Cheongju- and Takju- impregnation conditions [11] to find out the substance changes in the compounds such as 5-HMF(5-hydroxymethylfurfural), Catalpol, free sugar, crude fat, crude protein, crude fiber, harmful substances including Benzopyrene etc., and to identify content changes caused by steaming and drying[12-14]. Also, the drying temperature was set at $50^{\circ} \mathrm{C}$ in order to minimize the production of harmful substances and the variation of contents due to high-temperature drying.

\section{Materials and Methods}

\section{Materials and Reagents}

In this study, dried Rehmanniae Radix (Rehmanniae Radix Libosch, Kyoungbang Pharmacy, Incheon, South Korea) available at the market were used as samples, and the moisture content of the samples were measured to be within $10-11 \%$. The samples were taken out after being immersed in Takju (alcohol 5\%) or Cheongju (alcohol $15 \%$ ) for 24 hours, and they were steamed in an electric steamer (Tefal VC-102670) at a temperature of $98 \sim 100^{\circ} \mathrm{C}$ and dried at a temperature of $50^{\circ} \mathrm{C}$. The samples were compared with the control group of R.radix preparata (Kyoungbang Pharmacy, Incheon Korea) available at the market. Considering that they were natural products, we cut the specimens into $3 \mathrm{~cm}$ and mixed them well in order to reduce possible deviation errors, and $3 \mathrm{~kg}$ of the sample was used under each condition.

\section{General compositional analysis}

The general compounds of each powder prepared as samples by each round of streaming and drying were analyzed in accordance with the Korean Food Standards Codex. The moisture measurement was conducted using 1.1.1.1. Loss on Drying Test Method of 1.1.1. Moisture Test Method, Chapter 9 General Test Method in accordance with the Korean Food Standards Codex.

The content of ash was measured in accordance with 1.1.2 Ash Test Method of Chapter 7 General Test Method, the Food Standards Codex of the KFDA (September, 2013). The crude fat analysis was conducted in accordance with the crude fat analysis method of the Korean Food Standards Codex of the KFDA (September, 2013). The crude fiber analysis was carried out using the crude fiber test method of the Korean Food Standards Codex of the KFDA. The free sugar content was carried out using the sugar quality test method of the Korean Food Standards Codex of the KFDA (2009). $5 \mathrm{~g}$ of the sample was melted into $25 \mathrm{ml}$ of distilled water and then agitated in the water bath at $30^{\circ} \mathrm{C}$. After that, it was centrifuged at 3,000 rpm for 30 minutes, and acetonitrile was added to the supernatant to prepare $50 \mathrm{~mL}$ of the mixture. After passing the supernatant into $0.45 \mu \mathrm{m}$ membrane filter, it was analyzed using the HPLC, and Galactose, Glucose, Fructose, Sucrose, Maltose and Lactose(Sigma, St. Louis, MO, USA) were used as the standard substances. Carbohydrate analysis column $(4.6 \times 250 \mathrm{~mm}$, Waters, MA, USA) was used, and the column temperature was set at $30^{\circ} \mathrm{C}$. And the detection was carried out with the RID detector, and the flow rate was 1.0 $\mathrm{mL} / \mathrm{min}$ and the injection quantity was $20 \mu \ell[15]$.

\section{5-HMF and Catalpol Analysis}

After weighing approximately $0.1 \mathrm{~g}$ of uniformed Rehmanniae Radix powder, it was melted into $40 \mathrm{~mL}$ of $30 \%$ Methanol, and the mixture was centrifuged after ultrasonic extraction. After passing it through Syringe filter $(0.2 \mu \mathrm{m})$, it was used as the test solution and was analyzed with HPLC/UV Detector. The HPLC was Alliance 2695 HPLC system, Waters, USA; the ultrasonic cleaner 
was SD- $200 \mathrm{H}$ (Seong Dong Ultrasonics, Korea); the centrifugal separator was Legand Mach 1.6R (Thermo, Germany); $50 \mathrm{ml}$ tubes were used; the agitation was done at $4000 \mathrm{r} / \mathrm{min}$. For the preparation of the standard solutions, adequate amounts of Catalpol(Sigma, Cat.No. 50839) and 5-HMF(Sigma,Cat.No. H40807) were diluted with Methanol and used [16].

\section{Benzo(a)pyrene Analysis}

For the analysis on $\operatorname{Benzo}(\alpha)$ pyrene, samples were pretreated and the analysis was carried out using the UPLC/ FLD in accordance with the KFDA's Practical Guidelines for Analytical Methods of Benzo(a)pyrene (December 2008 and June 2011) and Benzo( $\alpha$ )pyrene Standards and Test Methods for Herbal Medicine (KFDA Notification No. 2009-13, April 12, 2009).

\section{Results and Discussion}

\section{Analysis Results of Free Sugar}

In this study, we used the traditional method of immersing dried Rehmanniae Radix in either Cheongju or Takju and steaming and drying and measured the contents of 6 kinds of free sugar depending on the repetition times of steaming and drying, and the results are as seen Fig. 1-6. For the dried Rehmanniae Radix available at the market, there was no detection of Galactose and Lactose, but traces of Fructose and Maltose were detected. For Cheongju immersion and steaming and drying, the contents of Galactose, Glucose and Fructose showed a sharp increase between the first round and third round of steaming and drying. In contrast, the content of Maltose showed a relatively smaller increase, but as the steaming and drying was repeated more, the content of Maltose was decreased sharply. There was no detection Cheongju-immersed samples after the 5th round and in Takju-immersed samples after the 7 th round. The content of Sucorse showed an increase after the second round of steaming and drying but a steep decline until the fourth round. The content of Galactose showed a significant increase after the first round of steaming and drying but a decrease after the third round. The contents of Glucose and Fructose showed a significant increase until the fourth round of steaming and drying but demonstrated little variation after the fifth round. The early content of Sucrose was the highest level, but the content of Glucose and Fructose showed an significantly increasing tendency as the process of steaming and drying was repeated more, and nine times steaming and nine times drying, they remained in large quantities in both Cheongju-immersed samples and Takju-immersed samples.

Compared to the samples steamed and dried after being immersed in Takju, those steamed and dried using Cheongju immersion showed increases in the contents of Galactose, Glucose and Sucrose but little increase in the content Maltose. The content of Sucrose showed an insignificant variation after the fifth round of steaming and drying. Depending on immersion methods and the repetition of steaming \& drying, the contents of Glucose and
Fructose showed significant increases. If we compare the contents of free sugar between Cheongju-immersed samples and Takju-immersed samples, those immersed in Cheongju showed a relatively higher content of free sugar after the seventh round of steaming and drying than those immersed in Takju.

\section{Analysis Results on 5-HMF and Catalpol}

In case of the dried Rehmanniae Radix distributed at the market, the content of Catalpol, a marker compound for Rehmanniae Radix, was found to be approximately 18 $\mathrm{mg} / \mathrm{g}$. As seen in Fig. 8, as the treatment temperature and time were increased, the content of Catalpol showed a rapidly decreasing tendency. After the fifth round of steaming and drying, Catalpol was not detected in both Cheongju-immersed samples and Takju-immersed samples. The study by Song et al. (2007) reported that in the event of steaming and drying at a high temperature of $110 \sim 120^{\circ} \mathrm{C}$, the content of Catalpol showed a steep decline after the fourth round, whereas in the event of drying at $50^{\circ} \mathrm{C}$ after being steamed at $98 \sim 100^{\circ} \mathrm{C}$, the content was reduced by half after the first round[4].

An experiment by Jeong, et al reported[17] that the halflife period of Catalpol was found to be after the fifth round of steaming and drying, which could be ascribed to steaming temperature. A decrease in Catapol content and an increase in 5-HMF content occurred at high temperatures. The dependence on temperature and time may be seen as the dependence on chemical reaction rather than enzymatic reaction. And, in order to increase the content of 5-HMF, it might be needed to consider increasing the content of sugar or applying the active titration temperature of glycolytic ferment additionally. The 50HMF content, a marker substance for Rehmanniae Radix endorsed by the KFDA, showed variations depending on the repetition frequency of steaming and drying as seen in Fig. 7.

After the first round of steaming and drying, 5-HMF was detected in both Takju-immersed samples and Cheongju-immersed samples. After the sixth round when there was no detection of Catalpol, the content of 5-HMF was found to be higher than $0.1 \%$, which is the content standard of the Korean Pharmacopoeia. There was no significant difference between Takju-immersed samples and Cheongju-immersed samples in terms of the content and pattern of 5-HMF production and drying but showed a rapid increase between the fifth and seventh round of steaming and drying but a slow increase after the seventh round, which proved the validity of the traditional 9 times steaming and 9 times drying method. The results of this study were similar to the study by Jeong et al.[6], which carried out a controlled trial of Takju and distilled water. For your reference, Rehmanniae radix preparata distributed at the market was found to have the 5-HMF content of $1.16 \mathrm{mg} / \mathrm{g}$, which was similar to the results of this study after the sixth round of steaming and drying, which met the content standard of the KFDA, but it was still lower than this study's content of approximately $3 \mathrm{mg} / \mathrm{g}$ after the ninth round of steaming and drying.

The results of changes in 5-HMF content that was produced due to a decrease in the concentration of free sugar 
seemed to be directly related to the study by Shin Sujeong et al. on the production of 5-HMF due to dehydration in fructose or glucose.

The heating-induced production of 5-HMF can occur not only in Rehmanniae radix preparata but also in garlic, and the content's increasing tendency depending on heating time was similar to Rehmanniae radix preparata, and the decreasing pattern of Sucrose during the production of 5HMF was also similar[18].

\section{Benzola)pyrene Analysis}

In the oriental medicine system of repeating the process of steaming and drying, natural drying has been used as the traditional drying method after steaming. In recent years, extreme temperature conditions are often set for the purpose of drying within a short time. If the drying temperature condition is too extreme, $\operatorname{Benzo}(\alpha)$ pyrene, a carcinogenic substance belonging to the polycyclic aromatic hydrocarbons (PAHs) group, can be produced. This substance is a crystalline solid which is produced by incomplete combustion at high temperatures. And it is present in coal tar, automobile exhaust gas, smoke from incinerated waste at inappropriate incineration facilities, tobacco smoke, etc., and due to environmental pollution, it is also present in uncooked or unprocessed food such as agricultural products, fish and shellfish. When food is cooked or processed using direct heat or smoke, carbohydrates, proteins, lipids, etc. can be dissolved or produced. The currently permitted $\operatorname{Benzo}(\alpha)$ pyrene content is " $5 \mu \mathrm{g}$ / kg or less" in case of Rehmanniae radix or Rehmanniae radix preparata ( $\ulcorner$ Benzo( $(\alpha)$ pyrene Standards and Test Methods for Herbal Medicine $\lrcorner$, KFDA Notification No. 2009-13, April 13, 2009).

Benzo( $\alpha$ )pyrene was not detected in the entire test group of Rehmanniae radix and also in the Rehmanniae radix preparata and dried Rehmanniae radix that were purchased from the market. Steaming at $98^{\circ} \mathrm{C}$ and drying at $50^{\circ} \mathrm{C}$ turned out to be a condition that had a very low chance of producing Benzo( $\alpha$ )pyrene, and, as expected, $\operatorname{Benzo}(\alpha)$ pyrene was not produced (Table 1).

\section{Changes in Ash, Crude Fat, Crude Protein, Crude Fiber Content}

As the steaming and drying process was repeated more, the content of ash did not show a significant change but the content of crude fiber showed an increasing tendency from $6.5 \%$ to $10.1 \%$, while the content of crude protein increased from $8.1 \%$ to $9.9 \%$. This is presumed to be due to the fact that the processes of Cheongju- or Takju-immersion, steaming and drying were repeated more, some fat-soluble and water-soluble compounds were eluted and, therefore, compounds with relatively insoluble material property remained. Especially after 3-4 rounds of steaming and drying, the content of crude fat did not show any increase in Cheongju-immersed samples but an increase in Takju-immersed samples. This is presumed to be due to that because Cheongju had a higher alcohol content than Takju, Cheongju-immersed samples did not show an increase in the content of crude fat after the steaming and drying process.

\section{Conclusion}

In the study, we carried out an analysis on changes in the content of Rehmanniae radix depending on each round of steaming and drying after immersing dried Rehmanniae radix in Takju and Cheongju, and the results are as follows. The contents of Galactose, Glucose and Fructose showed a rapid increase between the first round and the third round. In contrast, the contents of Maltose and Sucrose showed a sharp decline as the steaming and drying process was repeated more. Cheongju-immersed samples had no detection of Maltose after the fifth round, while Takju- immersed samples no detection of Maltose after the seventh round. In terms of the contents of Galactose and Glucose, Cheongju-immersed samples showed a slowdown in the decline of concentration after the fifth round, whereas Takju-immersed samples showed a slow concentration decrease after the seventh round.

The content of free sugar in Cheongju-immersed samples showed a constant level of concentration after the fifth round of steaming and drying, while Takju-immersed samples showed a constant tendency after the seventh round. As the steaming and drying was repeated more, the content of Catalpol showed a rapid decrease. The content of Catalpol was reduced by half in Takju-immersed samples and Cheongju-immersed samples only after the first round of steaming and drying but showed a slow decline from the second round to the fifth round. In order to increase the content of 5-HMF, it is necessary either to increase the content of sugar or to consider the active titration temperature for glycolytic ferment additionally. After the sixth round of steaming and drying where there was no detection of Catalpol, the content of 5-HMF was higher than the $0.1 \%$ content standard of the Korean Pharmacopoeia.

Although the content and pattern of 5-HMF did not show any significant difference between Takju-immersed samples and Cheongju-immersed samples, it showed a rapid increase between 5 to 7 rounds of steaming and drying. After the seventh round, it showed a relatively slower increase, which proved the validity of the traditional 9 times steaming and 9 times drying method. This is presumed to be due to the fact that as the processes of impregnation, steaming and drying are repeated more, some of the oil-soluble and water-soluble compounds were eluted and therefore, compounds with a relatively insoluble material property remained. These results can be useful foundation data for the validity of processing methods for Rehmanniae radix, and more in- depth and systematic studies need to be conducted on this issue.

\section{Acknowledgments}

This research was supported by grants from Hanseo University Research Foundation in 2017. 
Table 1 Comparison of 5-HMF, Catalpol, Benzo( $\alpha$ )pyrene contents between the market-distributed dried Rehmanniae radix and Rehmanniae radix preparata.

\begin{tabular}{|c|c|c|c|}
\hline Sample & $\begin{array}{l}5-\mathrm{HMF} \\
(\mathrm{mg} / \mathrm{g})\end{array}$ & $\begin{array}{l}\text { Catalpol } \\
(\mathrm{mg} / \mathrm{g})\end{array}$ & $\begin{array}{l}\text { Benzopyrene } \\
\quad(\mathrm{mg} / \mathrm{g})\end{array}$ \\
\hline $\begin{array}{c}\text { Dried Rehmanniae radix available at the } \\
\text { market } 1\end{array}$ & N/D & 19.29 & N/D \\
\hline $\begin{array}{c}\text { Dried Rehmanniae radix available at the } \\
\text { market } 2 \text { (Control) }\end{array}$ & $\mathrm{N} / \mathrm{D}$ & 17.87 & $\mathrm{~N} / \mathrm{D}$ \\
\hline $\begin{array}{c}\text { Rehmanniae radix preparata available at the } \\
\text { market } 1\end{array}$ & 1.16 & $\mathrm{~N} / \mathrm{D}$ & $\mathrm{N} / \mathrm{D}$ \\
\hline $\begin{array}{c}\text { After } 9 \text { times of Takju impregnation, steaming } \\
\text { and drying }\end{array}$ & 2.99 & N/D & N/D \\
\hline $\begin{array}{c}\text { After } 9 \text { times of Cheongju impregnation, } \\
\text { steaming and drying }\end{array}$ & 2.74 & $\mathrm{~N} / \mathrm{D}$ & N/D \\
\hline
\end{tabular}

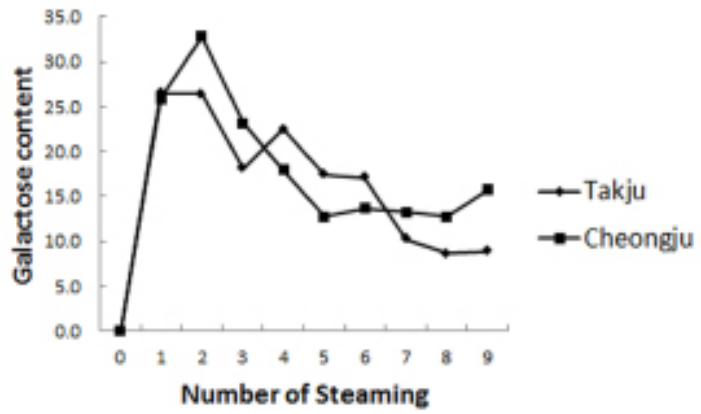

Fig 1. Variation in Galactose contents depending on the repetition frequency of steaming and drying

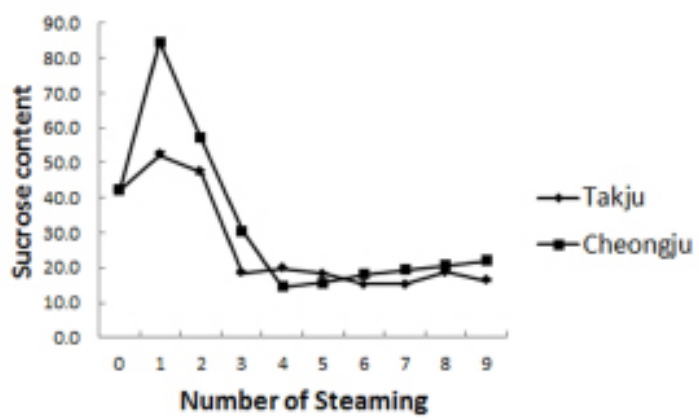

Fig. 3. Variation in Sucrose contents depending on the repetition frequency of steaming and drying.

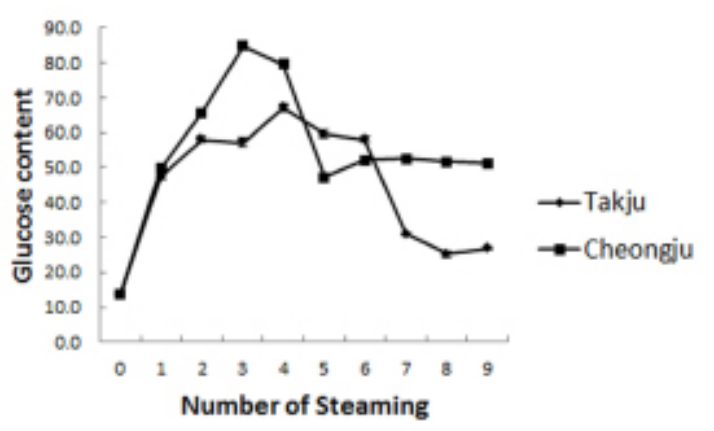

Fig. 2. Variation in Glucose contents depending on the repetition frequency of steaming and drying.

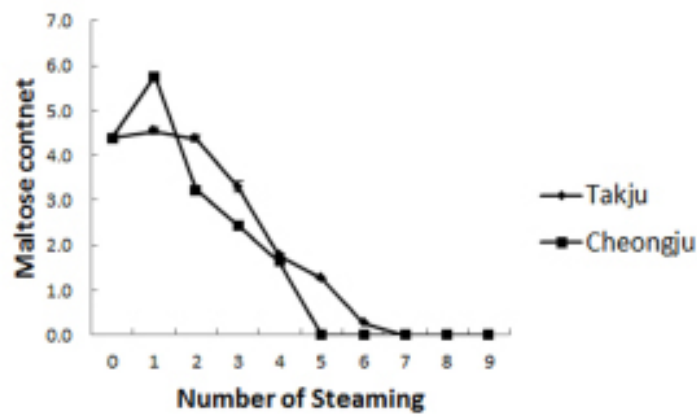

Fig. 4. Variation in Maltose contents depending on the repetition frequency of steaming and drying. 


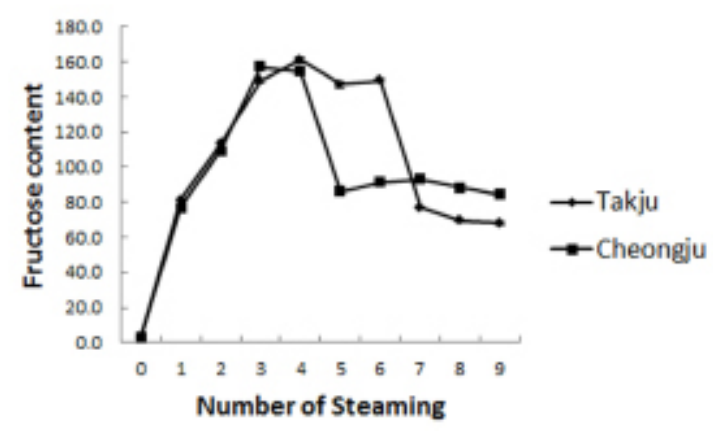

Fig 5. Variation in Fructose contents depending on the repetition frequency of steaming and drying.

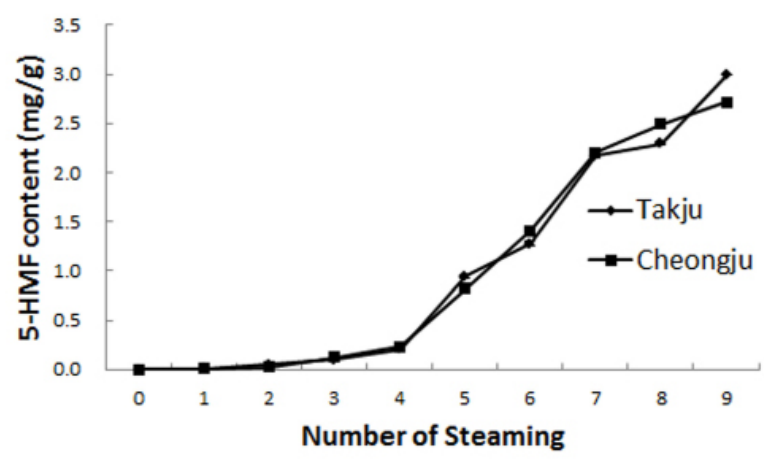

Fig 7. Variation in 5-HMF contents depending on the repetition frequency of steaming and drying.

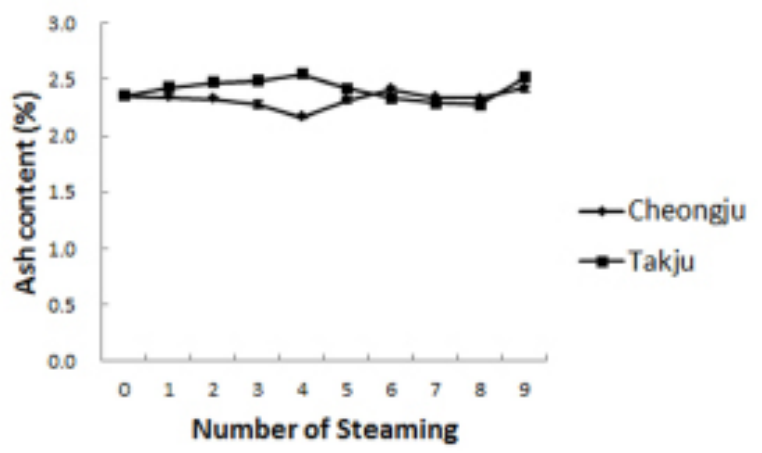

Fig 9. Variation in crude ash contents depending on the repetition frequency of steaming and drying.

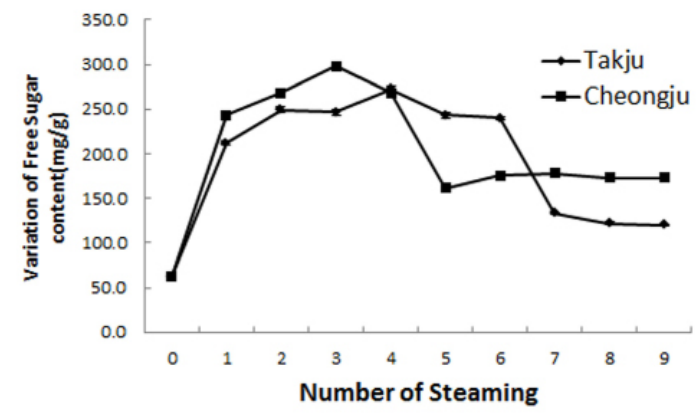

Fig 6. Variation in free sugar contents depending on the repetition frequency of steaming and drying.

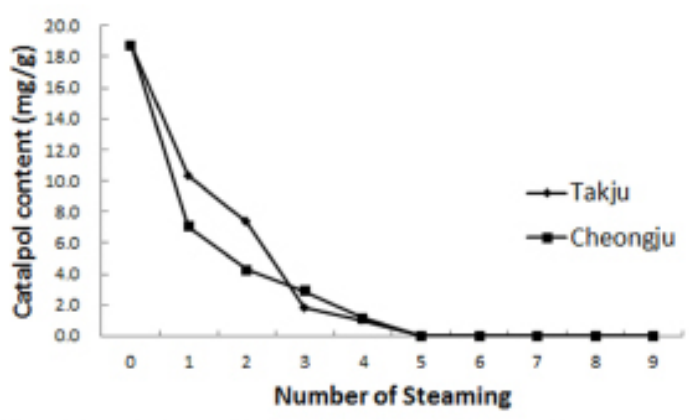

Fig 8. Variation in Catalpol contents depending on the repetition frequency of steaming and drying.

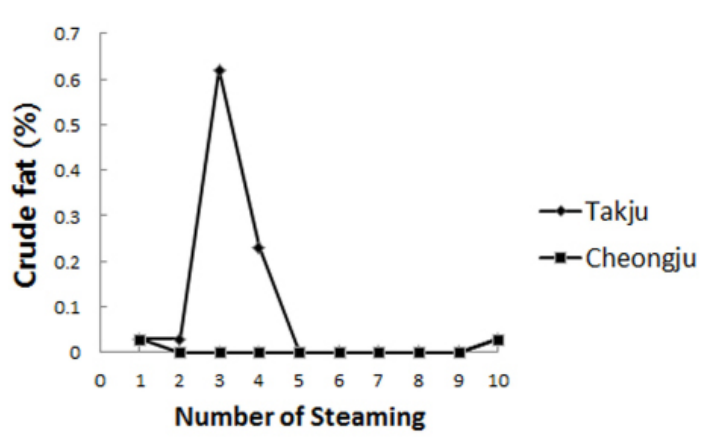

Fig 10. Variation in crude fat contents depending on the repetition frequency of steaming and drying. 


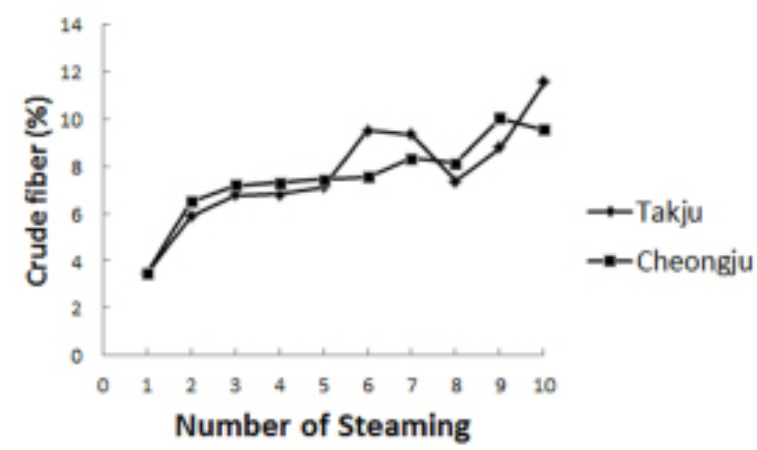

Fig 11. Variation in crude fiber content depending on the repetition frequency of steaming and drying.

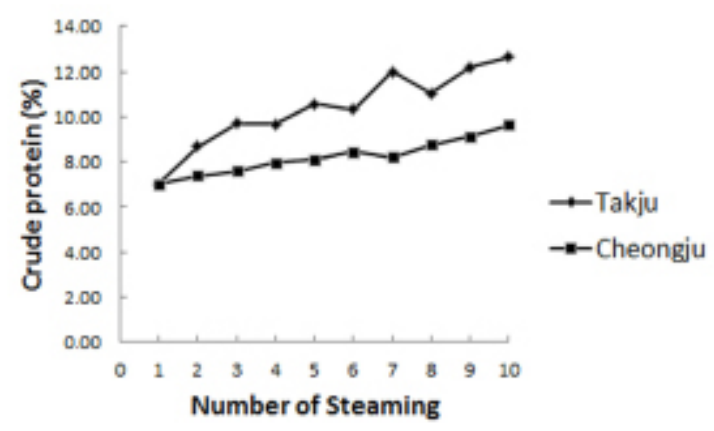

Fig 12. Variation in crude protein content depending on the repetition frequency of steaming and drying 


\section{References}

1. Han JH, Kim GH. Oriental Pharmacology. Euiseongdang Pub Co Seoul. 2008; 169.

2. Shin MG. Clinical Herbology (Colored version). Namseongdang Pub Co Seoul. 1986; 219.

3. Kang BS, Seo BI, Choi HY. Herbal Medicine Formulations and Clinical Applications. Younglimsa Pub Co Seoul. 2003; 205-10.

4. Song DS, Woo KS, Seong NS, Kim KY, Jeong HS, Lee HB. Changes in Quality of Rehmanniae radix Preparata with Heating Conditions J Korean Soc Food Sci Nutr. 2007; 36(6):773-8.

5. Woo KS, Song DS, Lee JS, Lee HB, Jeong HS. Quality Characteristics of Rehmannia radix Preparata with Pre-soaking Solvents Korean J Food Sci Technol. 2007; 39(3):289-94.

6. Jeong DJ, Seo YB. Experimental Studies on the Haematopoietic Effects of the Rehmanniae Radix Preparat. Kor J Herbology. 2001; 16(1):73-89.

7. Chang MS, Yang WM, Yu TW, Kim DR, Park EH, Ko EB, Choi MJ, Kim HY, Oh JH, Shim KJ, Yoon JW, Park SK. Antioxidant Effects of Rehmanniae Radix Preparat in GC-1 Cells. Kor J Herbology. 2007; 22(4):81-6.

8. Lee YB. Studies on the Effect of Sukjihwang on Dietary Hyperlipidemia and Antioxidant Activity. Journal of Environmental Science and Technology Research Center 2001; 11(1):41-57

9. Jin Y, Kim YJ, Jeon JN, Wang C, Min JW, Jung SY, Yang DC. Changes of Ginsenosides and Physiochemical Properties in Ginseng by New 9 Repetitive Steaming and Drying Process. Korean J Plant Res. 2012; 25(4):473-81

10. Lee CK, Seo JM. Changes of the consituents in the Rehmanniae radix preparata during processing. J Korean Soc Food Sci Nutr. 2004; 33:1748-52

11. Hwang SY, Hwang BY, Choi WH, Jung HJ, Huh JD, Lee KS, Ro JS. Quantitative determination of 5-hydroxymethyl-2-furaldehyde in the Rehmanniae radix Preparata samples at various processing stages. Kor J Pharmacogn. 2001; 32: 116-20.

12. Korean Association of Pharmacy Education compiled by Pharmacy Subcommittee. 8th Revision of Guide to the Korean Pharmacopoeia. Shinilsangsa Pub Co Seoul. 2003; 1166.

13. Zhu M, Hong SP, Kim CS, Lee JH. Determination Methods of Rehmanniae Radix by HPLC. Kor J Herbology. 2003; 18(3):203-9

14. Han JH, Park BR. Study on The Componential Changes of Rehmannia Radix Preparata by Steaming and Hotair Drying. Korean J Oriental Physiology \& Pathology. 2011; 25(5):823-9

15. Shih CK, Son YJ, Lee YJ. Changes in the carbohydrate contents of Rehmanniae radix during processing. Kor J Her. 1999; 14:1-11

16. Shin SJ. Quantitative Analysis of Reaction Products from Glucose and Xylose in Acidic Aqueous Medium By H-NMR Spectroscopic Method. J Korean Wood Sci \& Tech. 2013; 41(4):287-92

17. Lee JH, Koh JA, Hwang EY, Hong SP. Quantitative determination of 5-hydroxymethyl-2- furaldehyde from
Rehmanniae radix Preparata according to various processings. Kor J Her. 2002; 17(2):145-9

18. Woo KS, Song DS, Lee JS, Lee HB, Cho HS, Cho KJ, Cha JY, Yim JH, Kim JH. Quality Characteristics of Rehmannia radix Preparata with Pre-soaking Solvents. J Korean soc Food Sci Nutr. 2011; 40(1):84-8 